\title{
Modified Dunn Procedure is Superior to In Situ Pinning for Short-term Clinical and Radiographic Improvement in Severe Stable SCFE
}

\author{
Eduardo N. Novais MD, Mary K. Hill BA, \\ Patrick M. Carry BA, Travis C. Heare MD, \\ Ernest L. Sink MD
}

Received: 1 August 2014/Accepted: 4 December 2014/Published online: 12 December 2014

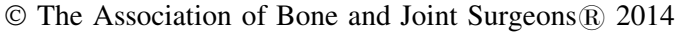

\begin{abstract}
Background In situ pinning is the conventional treatment for a stable slipped capital femoral epiphysis (SCFE). However, with a severe stable SCFE the residual deformity may lead to femoroacetabular impingement and articular cartilage damage. A modified Dunn subcapital realignment procedure has been developed to allow for correction at the level of the deformity while preserving the blood supply to the femoral head.

Questions/purposes We compared children with severe stable SCFE treated with the modified Dunn procedure or

Each author certifies that he or she, or a member of his or her immediate family, has no funding or commercial associations (eg, consultancies, stock ownership, equity interest, patent/licensing arrangements, etc) that might pose a conflict of interest in connection with the submitted article.

All ICMJE Conflict of Interest Forms for authors and Clinical Orthopaedics and Related Research ${ }^{\mathbb{R}}$ editors and board members are on file with the publication and can be viewed on request.

Clinical Orthopaedics and Related Research ${ }^{\circledR}$ neither advocates nor endorses the use of any treatment, drug, or device. Readers are encouraged to always seek additional information, including FDAapproval status, of any drug or device prior to clinical use. Each author certifies that his or her institution approved the human protocol for this investigation that all investigations were conducted in conformity with ethical principles of research, and that informed consent for participation in the study was obtained.

This study was performed at Children's Hospital Colorado, Aurora, CO, USA.
\end{abstract}

E. N. Novais ( $\square)$, M. K. Hill, P. M. Carry, T. C. Heare Department of Orthopaedic Surgery, Children's Hospital Colorado, 13123 E 16th Avenue, B060, Aurora, CO 80045, USA

e-mail: eduardo.novais@childrenscolorado.org

E. L. Sink

Department of Orthopaedic Surgery, Hospital for Special Surgery, New York, NY, USA in situ pinning in terms of (1) proximal femoral radiographic deformity; (2) Heyman and Herndon clinical outcome; (3) complication rate; and (4) number of reoperations performed after the initial procedure.

Methods In this nonmatched retrospective study, 15 patients treated with the modified Dunn procedure (between 2007 and 2012) and 15 treated with in situ pinning (between 2001 and 2009) for severe but stable SCFE were followed for a mean of 2.5 years (range, 1-6 years). During the period in question, the decision regarding which procedure to use was based on the on-call surgeon's discretion; six surgeons performed in situ pinning and three surgeons performed the modified Dunn procedure. A total of 15 other patients were treated for the same diagnosis during the study period but were lost to followup before 1 year; of those, 12 were in the in situ pinning group. Radiographs were reviewed to measure the AP and lateral alpha angles, femoral head-neck offset, and Southwick angle preoperatively and at the latest clinical visit. The Heyman and Herndon clinical outcome, complications, and subsequent hip surgeries were recorded.

Results At latest followup, the median AP alpha angle $\left(52^{\circ}\right.$, range $41^{\circ}-59^{\circ}$ versus $76^{\circ}$, interquartile range [IQR]: $\left.68^{\circ}-88^{\circ} ; \mathrm{p}=0.0017\right)$, median lateral alpha angle $\left(44^{\circ}\right.$, IQR: $40^{\circ}-51^{\circ}$ versus $87^{\circ}$, IQR: $\left.74^{\circ}-96^{\circ} ; \mathrm{p}<0.001\right)$, median head-neck offset (7 mm, IQR: 5-9 mm versus -5 , IQR: -11 to $-4 \mathrm{~mm}$; $\mathrm{p}<0.001)$, and median Southwick angle $\left(16^{\circ}\right.$, IQR: $6^{\circ}-23^{\circ}$ versus $58^{\circ}$, IQR: $47^{\circ}-66^{\circ}$; $\mathrm{p}<0.001)$ revealed better deformity correction with the modified Dunn procedure compared with in situ pinning. Nine patients had good or excellent results in the modified Dunn group compared with four of 15 in the in situ pinning group ( $\mathrm{p}=0.0343$; odds ratio, 5.86; 95\% CI, 1.13-40.43). With the numbers available, there were no differences in the numbers of complications in each group (five versus 
three complications in the in situ and modified Dunn groups, respectively; $p=0.66$ ), but there were more reoperations in the in situ pinning group (three versus seven; $\mathrm{p}=0.0230$ ).

Conclusions The modified Dunn procedure results in better morphologic features of the femur, a higher rate of good and excellent Heyman and Herndon clinical outcome, a lower reoperation rate, and a similar occurrence of complications when compared with in situ pinning for treatment of severe stable SCFE.

Level of Evidence Level III, therapeutic study.

\section{Introduction}

Treatment of slipped capital femoral epiphysis (SCFE) traditionally focused on stabilization of the physis by means of in situ pinning [3, 7-9]. Historically, in situ pinning was reported to provide good Iowa hip outcome scores at long-term followup [7, 8]. However, the longterm patient-reported outcome scores worsen and radiographic progression to osteoarthritis is more common with increasing severity of SCFE $[8,10,20]$. Although residual SCFE deformity may partially remodel after in situ pinning [29], the remodeling process leads to femoroacetabular impingement (FAI), an abnormal contact between the proximal femur and the anterior acetabular rim [30]. FAI secondary to SCFE has been reported to lead to articular cartilage damage $[1,21,22,32]$, which is related to the development of osteoarthritis [14].

Contemporary goals of surgical treatment of severe SCFE beyond stabilization of the physis include realignment of the proximal femur to restore the femoral head-neck anatomy and allow for better arc of hip motion, thereby avoiding FAI $[27,28]$. Corrective osteotomies performed at the subcapital level have the advantage of complete restoration of the femoral anatomy but are associated with potential risk of osteonecrosis of the femoral head [11,12]. Advancements in the understanding of the blood supply to the femoral head [15] and in the technique for safe dissection of the posterior retinaculum containing the nutrient vessels to the femoral head [13] have allowed for development of a modified Dunn subcapital realignment procedure using the surgical hip dislocation approach [24]. Encouraging results with complete restoration of proximal femoral anatomy and low rates of osteonecrosis for stable SCFE have been reported [17, 24, $26,33,39]$. Nevertheless, to our knowledge, only one study has evaluated the modified Dunn procedure versus in situ pinning for treatment of stable SCFE [34]. Souder et al. reported a $20 \%$ rate of osteonecrosis after the modified Dunn procedure for stable SCFE, however the severity of their cases was not reported [34].
In this study we compared children with severe stable SCFE treated with the modified Dunn procedure or in situ pinning in terms of (1) proximal femoral radiographic deformity; (2) Heyman and Herndon clinical outcome [16]; (3) complication rate; and (4) number of reoperations performed after the initial procedure.

\section{Patients and Methods}

After institutional review board approval, 323 patients who underwent treatment for SCFE between January 2001 and December 2012 were identified. Inclusion criteria were (1) diagnosis of severe (Southwick angle greater than $60^{\circ}$ on the frog lateral radiograph) [35, 36], stable (weightbearing with or without crutches at the time of presentation) [25] SCFE; and (2) a minimum of 1 year followup after initial surgery. We excluded 26 preslip hips, 154 with mild SCFE, 60 with moderate SCFE, 33 with unstable severe SCFE, and one hip that could not be classified owing to poor radiographic imaging. A total of 49 hips with severe stable slips remained. In cases of bilaterality $(n=2)$, only the first hip to undergo treatment was included. One patient was excluded owing to surgical treatment with a trochanteric femoral osteotomy and another resulting from highenergy trauma to the hip after initial SCFE treatment. Of the 45 remaining patients, 15 (33\%) were excluded because their followup was less than 1 year. Of the 15 patients lost to followup, 12 were in the in situ pinning group, one was in the modified Dunn group, and two had additional procedures at the time of surgery (one anterior-performed Fish osteotomy [12] and one femoral osteotomy). A total of 30 patients with a mean followup of 2.5 years (range, 1-6 years) were included. Fifteen patients underwent the modified Dunn procedure (between 2007 and 2012) and 15 had in situ pinning (between 2001 and 2009). The two groups were statistically comparable regarding age at surgery, sex, affected side, and duration of followup (Table 1).

During the study years, the decision regarding which procedure to use was based on the on-call surgeon's discretion based on the surgeon's familiarity with the approach; six surgeons performed only in situ pinning while three surgeons performed only the modified Dunn procedure. The modified Dunn procedure has been reported to allow for full restoration of femoral epiphyseometaphyseal alignment although it is a more technically demanding surgical procedure [17, 24, 26, 33, 39]. At our institution, since 2007 the modified Dunn procedure has been gradually favored for treatment of moderate and severe SCFE. There was an overlap of transition and since 2009 all patients with severe SCFE have been treated with the modified Dunn procedure by one of three surgeons 
Table 1. Patient characteristics by treatment group

\begin{tabular}{lccc}
\hline Variable & $\begin{array}{l}\text { In situ } \\
\text { pinning }\end{array}$ & $\begin{array}{l}\text { Modified Dunn } \\
\text { procedure }\end{array}$ & p value \\
\hline Sex & & & \\
$\quad$ Female, number (\%) & $6(40)$ & $4(27)$ & 0.439 \\
$\quad \begin{array}{l}\text { Male, number (\%) } \\
\text { Affected side }\end{array}$ & $9(60)$ & $11(73)$ & \\
$\quad \begin{array}{l}\text { Left, number (\%) } \\
\text { Right, number (\%) }\end{array}$ & $8(53)$ & $6(40)$ & 0.464 \\
$\begin{array}{l}\text { Age at surgery, } \\
\text { mean years (range) }\end{array}$ & $13(10-16)$ & $14(12-17)$ & 0.082 \\
$\begin{array}{l}\text { Followup, mean } \\
\text { years (range) }\end{array}$ & $2.5(1-6)$ & $2.4(1-5)$ & 0.825 \\
\hline
\end{tabular}

(ENN, ELS, TCH) who specialize in these procedures. A total of 15 other patients were treated for the same diagnosis during the study years but were lost to followup before 1 year; of those, 12 were in the in situ pinning group.

The medical records and radiographs of all eligible subjects were retrospectively studied. Medical records were assessed by one of the authors (MKH) not involved in the clinical care of the patients. All patients had experienced hip or thigh pain that had been aggravated by walking for more than 3 weeks. In all patients, the physical examination revealed a limp and obligatory external rotation with hip flexion. A pediatric orthopaedic fellow (JS) not involved in the' clinical care of the patients assessed preoperative and most recent AP and frog lateral radiographs of the pelvis. On the frog lateral view, slip severity was assessed by measurement of the Southwick angle [35, 36], and the contour of the femoral head-neck junction was assessed by measurement of the head-neck offset and modified alpha angle as previously described [23]. The alpha angle also was measured on the AP view. The two treatment groups were statistically comparable regarding severity of the preoperative deformity assessed by the AP alpha angle $(\mathrm{p}=0.1480)$, lateral alpha angle $(\mathrm{p}=$ 0.7336), lateral femoral head-neck offset $(p=0.9256)$, and Southwick angle $(\mathrm{p}=0.6143)$.

Treatment results in relation to pain, gait pattern, and hip motion at last visit or immediately before reoperation were assessed by a professional research assistant (MKH) using the Heyman and Herndon classification system [16]. According to this classification system, a hip is considered excellent if it has normal ROM, and there is no limp and no pain; good if there is no limp, no pain, and slight limitation of internal rotation but internal rotation beyond neutral; fair if there is no limp, no pain, and slight limitation of abduction and internal rotation; or poor if there is a mild limp, slight pain after strenuous exercise, and slight limitation of internal rotation, abduction, and flexion. A hip is considered failed if there is a limp, pain on activity, and marked limitation of motion requiring reconstructive surgery or progressive radiographic changes are seen. Treatment details, postoperative complications, and subsequent surgeries were recorded.

The modified Dunn procedure was performed by one of the three authors (ENN, ELS, TCH) according to a previously described technique [24]. Briefly, the patient is positioned in a lateral decubitus position and a lateral incision is performed. The fascia lata is split and the interval between the gluteus medius and maximus is dissected exposing the piriformis tendon. The interval between the piriformis and gluteus minimus is further dissected exposing the hip capsule posteriorly. The greater trochanter is cut with an oscillating saw to allow the piriformis tendon to remain attached to the stable portion of the trochanter. The displaced trochanteric fragment then is flipped anteriorly allowing for exposure of the hip capsule. An arthrotomy is performed in line with the femoral neck. The arthrotomy continues posteriorly toward the piriformis tendon in line with the acetabular rim and anteriorly toward the anteroinferior aspect of the acetabulum. The femoral head then is dislocated from the acetabulum by flexion and external rotation of the hip and the ligamentum teres is transected. The acetabular cavity is examined for chondrolabral disorders. The femoral head is reduced back in the acetabulum and the soft tissue retinaculum containing the nutrient vessels to the femoral head is dissected after the proximal portion of the stable trochanter is carefully removed [13]. The femoral neck is completely exposed after periosteal dissection. A curved osteotome is used as a lever to detach the femoral head from the physis and the posterior callus is trimmed from the metaphysis. Excessive shortening of the femoral neck should be avoided because of the risk of hip instability. The femoral head then is reduced back to the femoral neck in the anatomic alignment and fixed with either two 6.5-mm cannulated screws (Synthes, Inc, West Chester, PA, USA) or three 3.0-mm fully threaded wires. The periosteum is loosely reapproximated and the hip capsule closed. The trochanteric fragment is reduced to its bed and fixed anatomically with two or three $3.5-\mathrm{mm}$ screws (Synthes, Inc) using a compression technique. The wound is closed in layers. Postoperatively, patients follow a nonweightbearing protocol for approximately 6 weeks followed by protected weightbearing with crutches for 6 more weeks.

In situ pinning was performed by one of six different surgeons (ME, GG, FC, JR, NM, SC) with the patient on a radiolucent fracture table or a flat-topped radiolucent table. Briefly, an anterolateral skin incision is performed in the thigh. A guidewire is introduced in the anterior aspect of the femoral neck aiming toward the center of the femoral head in the AP and lateral projections. One $6.5-$ or $7.3-\mathrm{mm}$ 
cannulated screw (Synthes, Inc) then is placed, transfixing the femoral physis. Patients are allowed partial weightbearing with crutches for 6 weeks.

Descriptive statistics were used to summarize all variables. Point estimates for continuous variables were reported as the mean and SD when normally distributed and as the median and interquartile range when not normally distributed. Point estimates for categorical variables were reported as the frequency (number) and percentage. The demographics and clinical characteristics of the two groups were compared using t-tests and/or chi-square tests. Age was considered a potential confounding variable, and therefore was controlled for in the logistic regression models. Odds ratios (ORs) with 95\% CIs were estimated using multiple variable logistic regression analyses to compare the incidence of complications, need for subsequent surgery, and odds of a good or excellent Heyman and Herndon classification [16] between treatment groups. Wilcoxon rank-sum tests were used to compare betweengroup differences in the pre- and postoperative radiographic variables. Wilcoxon signed-rank tests were used to compare within-group changes (pre- versus postoperative measurements) in the radiographic variables.

\section{Results}

Femoral radiographic morphologic features were achieved more reliably in the modified Dunn group than the in situ pinning group. At latest followup, the median AP alpha angle $\left(52^{\circ}\right.$, interquartile range [IQR]: $41^{\circ}-59^{\circ}$ versus $76^{\circ}$, IQR: $\left.68^{\circ}-88^{\circ} ; \mathrm{p}=0.0017\right)$, the median lateral alpha angle (44, IQR: $40^{\circ}-51^{\circ}$ versus $87^{\circ}$, IQR: $74^{\circ}-96^{\circ} ; \mathrm{p}<0.001$ ) and the median Southwick angle $\left(16^{\circ}\right.$, IQR: $6^{\circ}-23^{\circ}$ versus $58^{\circ}$, IQR: $47^{\circ}-66^{\circ}$; $\left.\mathrm{p}<0.001\right)$ were lower in the modified Dunn group compared with the in situ pinning group. The median femoral head-neck offset also was better in the modified Dunn group ( $7 \mathrm{~mm}$, IQR: 5-9 mm versus -5, IQR: -11 to $-4 \mathrm{~mm} ; \mathrm{p}<0.001)$. The modified Dunn procedure resulted in improvement in all radiographic variables (Fig. 1). In the in situ group, all parameters except for the Southwick angle improved after surgery (Table 2).

Outcomes scores likewise favored the modified Dunn group. At latest followup, nine of 15 patients in the modified Dunn group had a good or excellent outcome according to the Heyman and Herndon classification [16] compared with four of 15 in the in situ pinning group $(\mathrm{p}=0.0343$; OR, 5.86; 95\% CI, 1.13-40.43) (Table 3).

With the numbers available, there were no differences in the numbers of complications in the groups. Three patients had five complications develop after in situ pinning, whereas two of 15 patients had three complications after the modified Dunn procedure $(\mathrm{p}=0.6588$; OR, $1.6 ; 95 \%$
Fig. 1A-F Radiographs from an 11-year-old girl with severe chronic stable SCFE treated by a modified Dunn procedure are shown. (A) The preoperative frog lateral radiograph shows abnormal femoral head-neck morphologic features with an increased alpha angle and reduced offset. (B) An AP radiograph taken preoperatively shows the severe deformity of the proximal femur. (C) An intraoperative photograph of the proximal aspect of the femur after surgical dislocation of the hip before development of the retinacular flap is shown. The anterior periosteum (black arrow) is torn and the metaphysis (asterisk) is prominent and severely displaced from the epiphysis. (D) An intraoperative photograph of the acetabulum shows articular cartilage damage extending from the superior to the anterior portion of the acetabular rim with chondral labral delamination (black arrow) and pitting malacia (white arrow). (E) The frog lateral radiograph taken at 2 years after surgery shows improved femoral head-neck offset and alpha angle. (F) An AP radiograph taken 2 years after surgery shows restored proximal femoral anatomy with normal alignment of the epiphysis in relation to the femoral neck.

CI, 0.20-15.03) (Table 4). No patients had chondrolysis of the hip.

Fewer unplanned reoperations were performed in patients treated with the modified Dunn procedure than patients treated with in situ pinning. In the modified Dunn group, two of 15 patients underwent three subsequent surgical procedures, including one revision of fixation owing to implant failure. One patient underwent removal of an intraarticular penetrating pin and subsequent THA for treatment of severe pain and deformity secondary to osteonecrosis. Seven patients in the in situ group underwent nine subsequent surgeries ( $p=0.0230$; OR, 8.4; 95\% CI, 1.32-90.37). Seven of these procedures, including femoral intertrochanteric osteotomy $(\mathrm{n}=3)$, surgical hip dislocation and femoral head-neck osteochondroplasty $(n=2)$, and surgical hip dislocation with a femoral head-neck osteochondroplasty and intertrochanteric osteotomy $(\mathrm{n}=2)$, were performed for the treatment of FAI-related pain and limited motion of the hip affecting activities of daily life. One patient experienced slip progression despite in situ pinning and underwent revision of fixation with one additional cannulated screw. He later had osteonecrosis develop and underwent an intertrochanteric osteotomy because of pain and abnormal hip motion (Fig. 2).

\section{Discussion}

Severe SCFE results in major deformity of the proximal femur and abnormal hip mechanics leading to FAI and articular cartilage damage [21, 22, 32], which is associated with premature osteoarthritis [1]. Despite historically better long-term outcomes after in situ pinning compared with reorientation procedures for mild and moderate SCFE, the residual deformity in severe SCFE is recognized to negatively affect its prognosis [7, 8]. The modified Dunn 

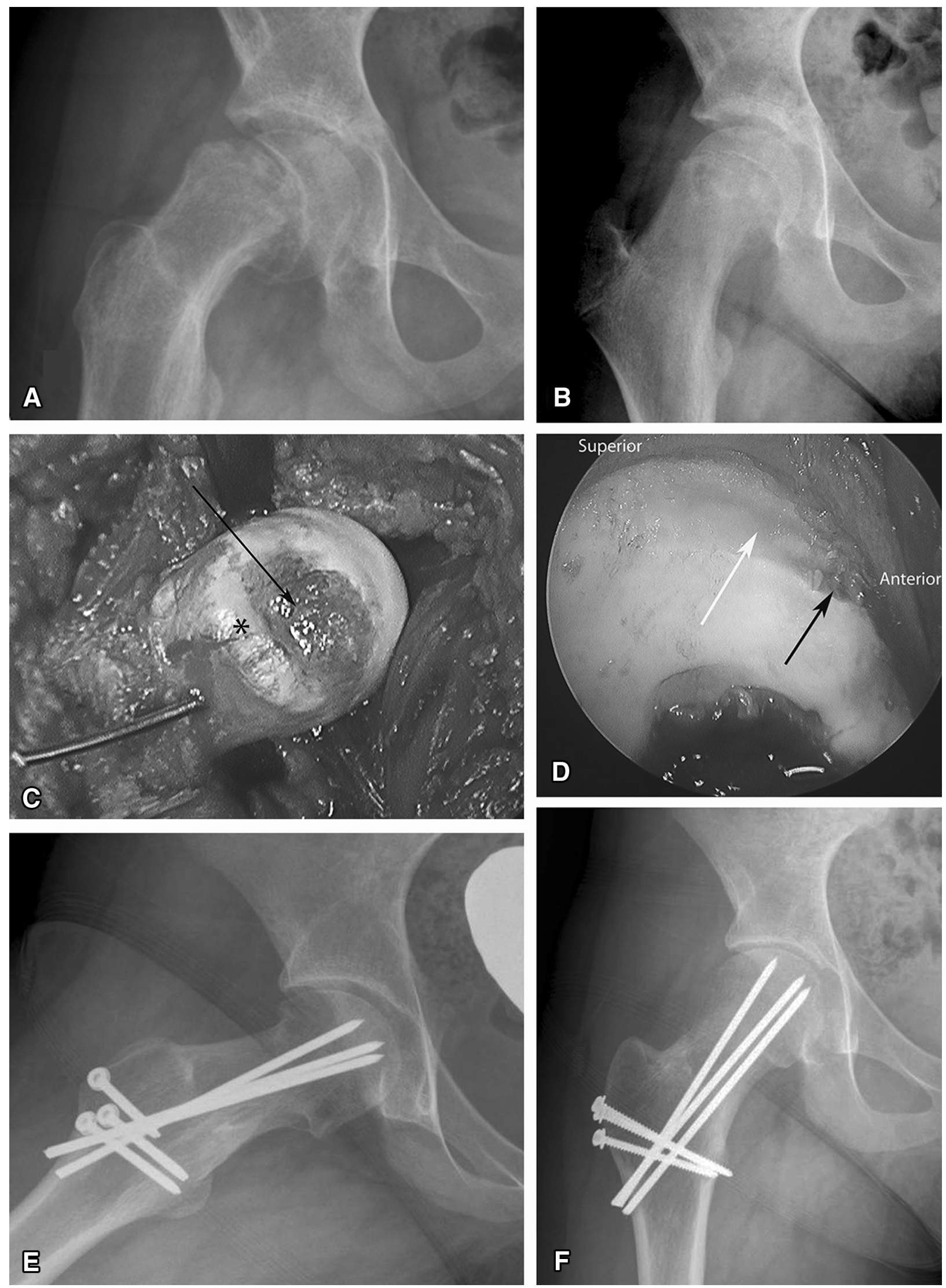
Table 2. Pre- and postoperative radiographic parameters by treatment group

\begin{tabular}{lcllllll}
\hline Parameter, median (interquartile range) & In situ pinning & & & \multicolumn{3}{l}{ Modified Dunn procedure } \\
\cline { 2 - 3 } & Preoperative & Postoperative & p value* & & Preoperative & Postoperative & p value* \\
\hline AP $\alpha$ angle (degrees) & $85(77-91)$ & $76(68-88)$ & 0.037 & $96(85-103)$ & $52(41-59)$ & $<0.001$ \\
Lateral $\alpha$ angle (degrees) & $106(95-113)$ & $87(74-96)$ & $<0.001$ & $111(93-120)$ & $44(40-51)$ & $<0.001$ \\
Femoral head-neck offset $(\mathrm{mm})$ & $-11(-13$ to -8$)$ & $-5(-11$ to -4$)$ & 0.019 & $-11(-18$ to -7$)$ & $7(5-9)$ & $<0.001$ \\
Southwick angle (degrees) & $63(60-74)$ & $58(47-66)$ & 0.142 & $65(54-81)$ & $16(6-23)$ & $<0.001$ \\
\hline
\end{tabular}

* $\mathrm{p}$ values correspond to within-group changes in radiographic measurements based on the Wilcoxon rank-sum test.

Table 3. Heyman and Herndon classifications by treatment group

\begin{tabular}{llllll}
\hline Treatment group & Failure & Poor & Fair & Good & Excellent \\
\hline $\begin{array}{l}\text { In situ pinning, number }(\%) \\
7(47)\end{array}$ & $3(20)$ & $1(7)$ & $1(7)$ & $3(20)$ \\
$\begin{array}{c}\text { Modified Dunn procedure, } \\
\text { number }(\%)\end{array}$ & $1(7)$ & $5(33)$ & 0 & $2(13)$ & $7(47)$ \\
\hline
\end{tabular}

Table 4. Postoperative complications by treatment group

\begin{tabular}{llllll}
\hline Complication type & \multicolumn{2}{l}{ In situ pinning } & & \multicolumn{2}{l}{$\begin{array}{l}\text { Modified Dunn } \\
\text { procedure }\end{array}$} \\
\cline { 2 - 3 } \cline { 5 - 6 } \cline { 5 - 6 } & Number & Percent & & Number & Percent \\
\hline Osteonecrosis & 1 & 7 & & 1 & 7 \\
Slip progression & 1 & 7 & & 0 \\
Pin impingement & 2 & 13 & & 0 & 0 \\
Implant failure & 0 & 0 & & 7 & 7 \\
Intraarticular pin penetration & 0 & 0 & 1 & 7
\end{tabular}

subcapital realignment procedure using a surgical hip dislocation approach has been reported to provide restoration of the femoral head-neck anatomy with low rates of osteonecrosis for treatment of stable SCFE [17, 24, 26, 33, 39]. Despite the reported low rates of osteonecrosis, the modified Dunn technique may have a higher risk when compared with in situ pinning. Moreover, because of the learning curve associated with the technically demanding modified Dunn procedure, the decision between its use versus in situ pinning often is based on surgeon experience rather than available evidence. In this study, we compared the modified Dunn procedure with in situ pinning regarding restoration of the proximal femoral anatomy, Heyman and Herndon clinical outcome [16], complication rate, and frequency of subsequent surgical interventions after treatment of severe stable SCFE.

Our study has numerous limitations. First, by including only patients with severe stable SCFE, we limited the number of patients in each treatment group. However, severe stable SCFE is rare. In the consecutive multicenter series by Ziebarth et al. [39], there were only five stable SCFE cases with documented preoperative Southwick angles greater than $60^{\circ}$. To our knowledge, our study is the largest series specifically evaluating severe stable SCFE and we believe the addition of a comparison group strengthens our findings. With the numbers available there was no difference in the complication rate between the two groups. However, we acknowledge that this study may have been underpowered to definitively draw this conclusion and further investigation is recommended. Second, potential assessment bias existed because the radiographic measurements and Heyman and Herndon classification [16] were determined unblinded to treatment. We attempted to control for this by using assessors not involved in the subjects' clinical care. Third, the retrospective nature of this study did not allow for standardization of surgical technique in the in situ group. Six experienced pediatric orthopaedic surgeons performed all the in situ pinning procedures and the modified Dunn procedures were performed by one of three of the authors using a more standardized technique. This raises a concern for selection bias, especially in the transition time where both treatments were performed at our institutions. It may have been that more complex cases were referred to one of the three surgeons performing the modified Dunn procedure while other not-so-involved cases were treated by the on-call surgeon by in situ pinning. Although we did not compare two sequential series in this study, the modified Dunn technique has been used only since 2007 and all patients treated by in situ pinning were treated before 2009. Fourth, our specific goal in this study was to comparatively investigate in situ pinning and the modified Dunn procedure as definitive treatment modalities for severe stable SCFE. We found that $1 / 2$ of the patients in the in situ pinning group underwent treatment for persistent hip pain related to the femoral deformity and FAI. Therefore, in situ pinning alone may not be sufficient to allow complete resolution of symptoms and improve function and hip motion in patients with severe stable SCFE. However, other surgical options outside the scope of this study exist, including different osteotomies performed at the base of the femoral neck $[5,19]$ or at the subtrochanteric level $[18$, $31,35,36]$. These procedures are not as technically demanding as the modified Dunn approach and one potential strategy to manage severe stable SCFE could 

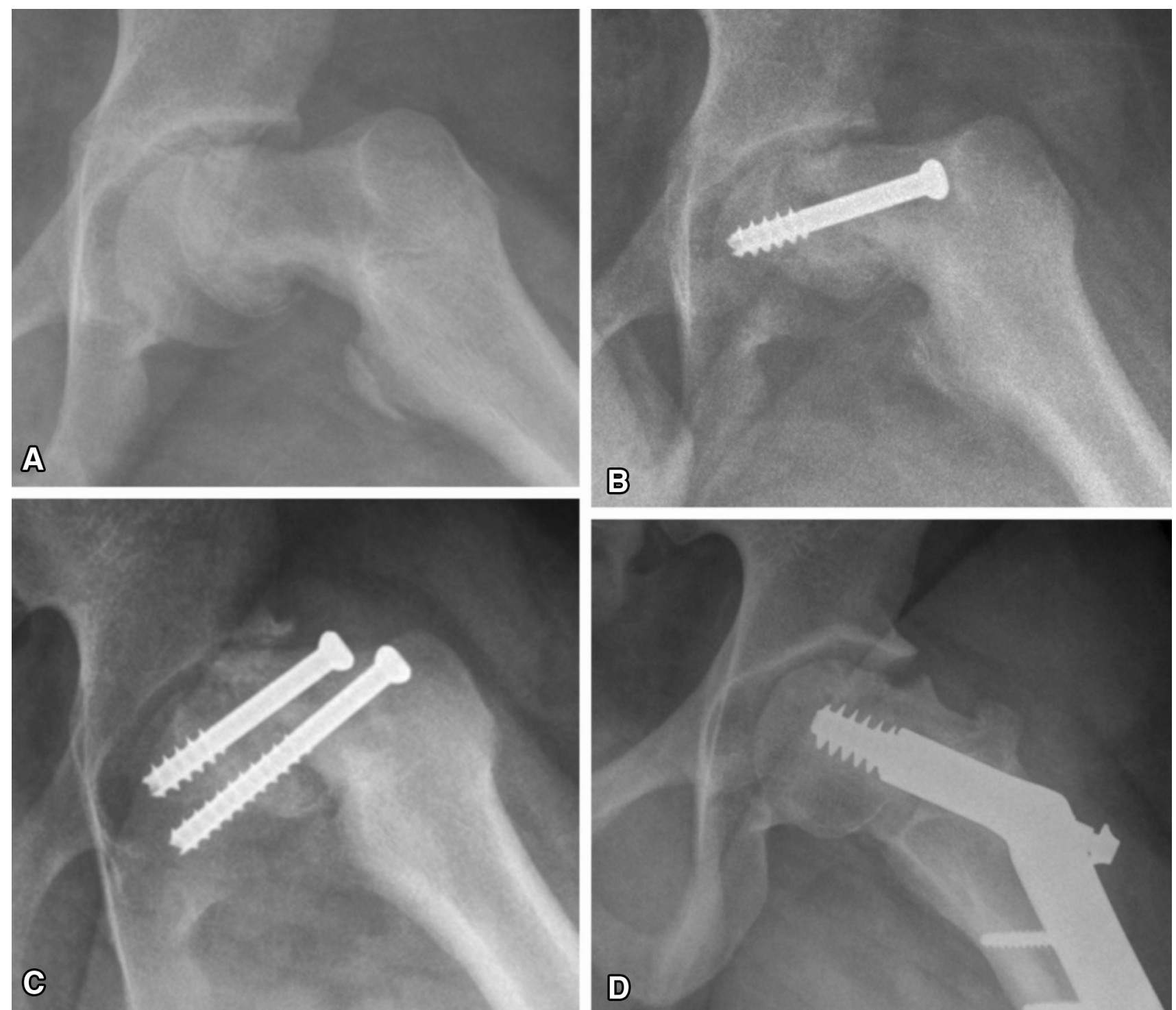

Fig. 2A-D Radiographs from a 13-year-old boy with severe stable SCFE treated by in situ fixation with an additional complication of progressive slip and osteonecrosis of the femoral head are shown. (A) The preoperative frog lateral radiograph shows a severe SCFE of the left hip. (B) The radiograph taken 2 months after in situ pinning shows progression of the slip and inadequate position of the screw in relation to the center of the femoral head. (C) The patient underwent revision of the fixation with an additional screw. The lateral

involve in situ pinning followed by a staged, planned femoral osteotomy.

Another source of limitation is the $70 \%$ followup rate of our series. Of the 15 patients lost to followup 12 were in the in situ pinning group, one in the modified Dunn group, and two had additional procedures at the time of surgery (one anterior-performed Fish osteotomy [12] and one femoral osteotomy). We acknowledge the potential source of transfer bias owing to the unequal loss to followup, which is attributable to the modified Dunn and the

radiograph shows lucency in the femoral head with sclerosis on the acetabular rim. (D) The frog lateral radiograph taken 4 years after a proximal femoral valgus flexion derotational osteotomy shows the femoral head has lost its sphericity secondary to osteonecrosis and there is a residual deformity of the femoral head and neck junction with a large prominence. The patient has a mild limp, mild pain exacerbated by activities, and limited flexion, internal rotation, and abduction of the left hip compared with the contralateral normal hip.

in situ pinning procedures requiring different followup regimens. Because patients who have in situ pinning often require fewer weightbearing restrictions and precautions during the immediate postoperative period, they may be less likely to be followed up for a long time. However, the concerns regarding subcapital realignment healing, longer rehabilitation process, and the potential for osteonecrosis make patients in the modified Dunn group more likely to return for postoperative evaluation. Likewise, we acknowledge that the difference in 
postoperative protocol is a concern for cointervention bias. The longer restriction on weightbearing was different between the two groups and it could have affected the short-term results evaluated in this study. Finally, another concern regarding the unequal loss of followup in the in situ pinning group is that the patients who were lost to followup could have had different outcomes than those who completed the study. The patients who were treated by in situ pinning may not have returned for followup because they could have had a bad clinical outcome and went elsewhere for treatment. Finally, the short-term followup may have not allowed capture of all potential complications associated with severe SCFE in the long term, including degenerative osteoarthritis. Despite these concerns, the length of followup was similar between the two treatment groups.

Complete restoration of the femoral head-neck anatomy after the modified Dunn procedure was not unforeseen [26, $33,39]$. However, to our knowledge, this is the first study to show a difference in the Southwick angle, alpha angle, and femoral head-neck offset between the modified Dunn procedure and in situ pinning for treatment of severe SCFE. In our series, the median alpha angle on the lateral frog radiographic view after the modified Dunn procedure was $44^{\circ}$, which is in line with previous studies in which mean lateral alpha angles between $40.6^{\circ}$ and $46^{\circ}$ were reported [2, $26,38]$. The median lateral alpha angle of $87^{\circ}$ after in situ pinning was greater than that in the modified Dunn group and greater than reported reference normal values of $42^{\circ}$ and $47^{\circ}$ in female and male adolescents, respectively [6]. The alpha angle, however, was substantially improved at most recent followup in the in situ pinning group. We attribute this improvement to femoral head-neck junction remodeling after in situ pinning [29]. The remodeling process is a result of repetitive impingement of the prominent metaphyseoepiphyseal junction into the acetabulum that leads to cartilage damage [30]. However, this process in patients with severe SCFE is unlikely to fully restore the proximal femoral anatomy. Castaneda et al. [10] reported that after a mean of 22 years after in situ pinning, $80 \%$ of patients had a pistol grip deformity and all patients had radiographic signs of osteoarthritis. Wensaas et al. [37] reported on 36 patients followed for an average of 37 years after in situ pinning and reported that persistent deformity with radiologic FAI is associated with poorer clinical and radiologic long-term outcome. In our study, the proximal femur deformity was corrected after a modified Dunn procedure but not after in situ pinning. However, with the short-term followup in our study, we were unable to determine if restoration of the femoral anatomy after the modified Dunn procedure allows for long-term joint survival.

Our findings suggest less hip pain and better motion and walking ability in the short term after the modified Dunn procedure compared with in situ pinning. The clinical outcome scores after the modified Dunn procedure are in line with those of Masse et al. [26], who reported a mean Harris hip score of 98.2 of 100 points. The main limitation in six of their patients was occasional mild pain, which would correspond to a poor result according to the Heyman and Herndon classification [16]. In our series, we found a very low rate of good or excellent outcomes [16] after in situ pinning. In a series of 105 patients with severe stable SCFE treated by in situ pinning and followed for a mean of 5.5 years, Castaneda et al. [9] reported that $76 \%$ achieved good or excellent results as assessed by the Iowa Hip Score. Larson et al. [20] reported on 176 hips with patients having a mean followup of 16 years after SCFE. Twelve percent of their patients underwent reconstructive surgery and an additional $33 \%$ experienced persistent pain after in situ pinning [20]. We believe surgical correction of the deformity with a modified Dunn procedure may be considered because of the relative high rate of unsatisfactory clinical results after in situ pinning in patients with severe SCFE.

With the numbers available, we found no difference in the odds of complications developing after in situ pinning versus the modified Dunn procedure. Osteonecrosis of the femoral head is the most worrisome complication after SCFE treatment. One of our patients (7\%) had osteonecrosis develop after in situ pinning. Although rare, osteonecrosis has been reported in as much as $5 \%$ of severe slips treated by in situ pinning [9]. Similar to Castaneda et al. [9], we attribute the osteonecrosis after in situ pinning in our patient to an inadequate cannulated screw position and slip progression after fixation. In addition, one of our patients (7\%) had osteonecrosis develop after the modified Dunn procedure. Slongo et al. [33] also reported only one osteonecrosis occurrence in nine severe SCFE cases treated by the modified Dunn procedure. Ziebarth et al. [39] reported no cases of osteonecrosis in five patients with severe stable SCFE. Masse et al. [26] also reported no cases of osteonecrosis in five patients with severe stable SCFE. We are aware of only one comparative study between in situ pinning and the modified Dunn procedure [34]. In a retrospective series by Souder et al. [34], 64 patients with stable SCFE were treated with in situ pinning and 10 had the modified Dunn procedure. They concluded that in situ pinning was substantially safer regarding osteonecrosis (no osteonecrosis after in situ pinning; two of 10 patients had osteonecrosis develop after the modified Dunn procedure; $p=0.02$ ). However, there was no stratification of SCFE severity in their study. Furthermore, similar to our findings, they reported that there was no clear relationship between complication rate and treatment method. Osteonecrosis of the femoral head, however, is the most important complication of SCFE treatment that is associated with poor long-term hip function 
and early degenerative joint changes. Future studies should investigate whether specific patient characteristics, including the severity of the femoral neck adaptive deformity and the growth plate status, play a role in the rate of osteonecrosis or if it is directly related to only surgeons' experience with the procedure.

Our data suggest that the modified Dunn procedure is more reliable than in situ pinning in terms of definitive treatment. Half of the patients treated by in situ pinning underwent additional surgical intervention owing to hip pain and limited motion associated with FAI. Arora et al. [4] also reported a high rate (77\%) of secondary surgery within 4 years after in situ pinning. In contrast, Souder et al. [34] found no significant relationship between reoperation rate and treatment method. They recommended in situ pinning to minimize risk of osteonecrosis but acknowledged that patients might need additional treatment for residual FAI deformity. In our series, $13 \%$ (two of 15) of patients in the modified Dunn group required implant revision because of failure. The ideal implant for fixation after the modified Dunn procedure has not been well established. Huber et al. [17] reported a $13 \%$ rate of implant failure requiring revision, whereas Ziebarth et al. [39] reported a $7.5 \%$ rate. Although our study averaged 2 years of followup, we believe it captured most potential short and mid-term complications after the modified Dunn procedure that potentially could require surgical treatment. The modified Dunn procedure allowed for restoration of the morphologic features of the proximal femur as seen by the radiographic analysis, however it does not preclude that these hips will not have secondary osteoarthritis develop. Long-term studies of patients undergoing the modified Dunn procedure for treatment of severe stable SCFE should be done to determine whether radiographic restoration of morphologic features of the femur avoids osteoarthritis of the hip.

We found that the modified Dunn subcapital realignment procedure using a surgical hip dislocation approach allows for better radiographic correction of the femoral head and neck deformity, better clinical Heyman and Herndon outcome classification [16], and lower reoperation rate when compared with in situ pinning for treatment of severe stable SCFE at an average of 2 years of followup. Moreover, there was a relative low rate of complications after the modified Dunn procedure, however the surgeons performing the procedures were experienced and it is unclear whether our results would be reproduced by surgeons with less experience. In situ pinning is less technically challenging, however it is offset by the lack of deformity correction and increased risk of secondary procedures for treatment of residual FAI after severe SCFE. The orthopaedic surgeon should assess these risks on an individual basis to allow for informed decision with patients. Radiographic correction after the modified Dunn procedure is impressive, however more studies are needed to evaluate whether restoring the proximal femur anatomy plays a role in preventing the development of osteoarthritis associated with severe stable SCFE.

Acknowledgments We thank Joseph Stone MD, (Pediatric Orthopaedics Associates, Atlanta GA, USA) for assistance with radiograph measurements. We acknowledge the six surgeons whose patients were included in the study: Mark Erickson MD, Gaia Georgopolous MD, Frank Chang MD, Nancy Miller MD, PhD, Jason Rhodes MD, and Steven Conlan MD (all from the Department of Orthopaedic Surgery, Children's Hospital Colorado Aurora, CO, USA).

\section{References}

1. Abraham E, Gonzalez MH, Pratap S, Amirouche F, Atluri P, Simon P. Clinical implications of anatomical wear characteristics in slipped capital femoral epiphysis and primary osteoarthritis. $J$ Pediatr Orthop. 2007;27:788-795.

2. Anderson LA, Gililland JM, Pelt CE, Peters CL. Subcapital correction osteotomy for malunited slipped capital femoral epiphysis. J Pediatr Orthop. 2013;33:345-352.

3. Aronson DD, Peterson DA, Miller DV. Slipped capital femoral epiphysis: the case for internal fixation in situ. Clin Orthop Relat Res. 1992;281:115-122.

4. Arora S, Dutt V, Palocaren T, Madhuri V. Slipped upper femoral epiphysis: outcome after in situ fixation and capital realignment technique. Indian J Orthop. 2013;47:264-271.

5. Barmada R, Bruch RF, Gimbel JS, Ray RD. Base of the neck extracapsular osteotomy for correction of deformity in slipped capital femoral epiphysis. Clin Orthop Relat Res. 1978;132:98101.

6. Bixby SD, Kienle KP, Nasreddine A, Zurakowski D, Kim YJ, Yen YM. Reference values for proximal femoral anatomy in adolescents based on sex, physis, and imaging plane. Am J Sports Med. 2013;41:2074-2082.

7. Boyer DW, Mickelson MR, Ponseti IV. Slipped capital femoral epiphysis: long-term follow-up study of one hundred and twentyone patients. J Bone Joint Surg Am. 1981;63:85-95.

8. Carney BT, Weinstein SL, Noble J. Long-term follow-up of slipped capital femoral epiphysis. J Bone Joint Surg Am. 1991;73:667-674.

9. Castaneda P, Macias C, Rocha A, Harfush A, Cassis N. Functional outcome of stable grade III slipped capital femoral epiphysis treated with in situ pinning. J Pediatr Orthop. 2009;29:454-458.

10. Castaneda P, Ponce C, Villareal G, Vidal C. The natural history of osteoarthritis after a slipped capital femoral epiphysis/the pistol grip deformity. J Pediatr Orthop. 2013;33(suppl 1):S76-82.

11. Dunn DM. The treatment of adolescent slipping of the upper femoral epiphysis. J Bone Joint Surg Br. 1964;46:621-629.

12. Fish JB. Cuneiform osteotomy of the femoral neck in the treatment of slipped capital femoral epiphysis: a follow-up note. $J$ Bone Joint Surg Am. 1994;76:46-59.

13. Ganz R, Huff TW, Leunig M. Extended retinacular soft-tissue flap for intra-articular hip surgery: surgical technique, indications, and results of application. Instr Course Lect. 2009;58:241-255.

14. Ganz R, Parvizi J, Beck M, Leunig M, Notzli H, Siebenrock KA. Femoroacetabular impingement: a cause for osteoarthritis of the hip. Clin Orthop Relat Res. 2003;417:112-120. 
15. Gautier E, Ganz K, Krugel N, Gill T, Ganz R. Anatomy of the medial femoral circumflex artery and its surgical implications. $J$ Bone Joint Surg Br. 2000;82:679-683.

16. Heyman $\mathrm{CH}$, Herndon $\mathrm{CH}$. Epiphyseodesis for early slipping of the upper femoral epiphysis. J Bone Joint Surg Am. 1954;36:539555.

17. Huber H, Dora C, Ramseier LE, Buck F, Dierauer S. Adolescent slipped capital femoral epiphysis treated by a modified Dunn osteotomy with surgical hip dislocation. J Bone Joint Surg Br. 2011;93:833-838.

18. Imhauser G. [Late results of Imhauser's osteotomy for slipped capital femoral epiphysis (author's transl)] [in German]. Z Orthop Ihre Grenzeb. 1977;115:716-725.

19. Kramer WG, Craig WA, Noel S. Compensating osteotomy at the base of the femoral neck for slipped capital femoral epiphysis. $J$ Bone Joint Surg Am. 1976;58:796-800.

20. Larson AN, Sierra RJ, Yu EM, Trousdale RT, Stans AA. Outcomes of slipped capital femoral epiphysis treated with in situ pinning. J Pediatr Orthop. 2012;32:125-130.

21. Leunig M, Casillas MM, Hamlet M, Hersche O, Notzli H, Slongo T, Ganz R. Slipped capital femoral epiphysis: early mechanical damage to the acetabular cartilage by a prominent femoral metaphysis. Acta Orthop Scand. 2000;71:370-375.

22. Leunig M, Fraitzl CR, Ganz R. [Early damage to the acetabular cartilage in slipped capital femoral epiphysis: therapeutic consequences] [in German]. Orthopade. 2002;31:894-899.

23. Leunig M, Horowitz K, Manner H, Ganz R. In situ pinning with arthroscopic osteoplasty for mild SCFE: a preliminary technical report. Clin Orthop Relat Res. 2010;468:3160-3167.

24. Leunig M, Slongo T, Kleinschmidt M, Ganz R. Subcapital correction osteotomy in slipped capital femoral epiphysis by means of surgical hip dislocation. Oper Orthop Traumatol. 2007;19:389-410.

25. Loder RT, Richards BS, Shapiro PS, Reznick LR, Aronson DD. Acute slipped capital femoral epiphysis: the importance of physeal stability. J Bone Joint Surg Am. 1993;75:1134-1140.

26. Masse A, Aprato A, Grappiolo G, Turchetto L, Campacci A, Ganz R. Surgical hip dislocation for anatomic reorientation of slipped capital femoral epiphysis: preliminary results. Hip Int. 2012;22:137-144.
27. Millis MB, Novais EN. In situ fixation for slipped capital femoral epiphysis: perspectives in 2011. J Bone Joint Surg Am. 2011;93(suppl 2):46-51.

28. Novais EN, Millis MB. Slipped capital femoral epiphysis: prevalence, pathogenesis, and natural history. Clin Orthop Relat Res. 2012;470:3432-3438.

29. O'Brien ET, Fahey JJ. Remodeling of the femoral neck after in situ pinning for slipped capital femoral epiphysis. J Bone Joint Surg Am. 1977;59:62-68.

30. Rab GT. The geometry of slipped capital femoral epiphysis: implications for movement, impingement, and corrective osteotomy. J Pediatr Orthop. 1999;19:419-424.

31. Schai PA, Exner GU, Hansch O. Prevention of secondary coxarthrosis in slipped capital femoral epiphysis: a long-term followup study after corrective intertrochanteric osteotomy. $J$ Pediatr Orthop B. 1996;5:135-143.

32. Sink EL, Zaltz I, Heare T, Dayton M. Acetabular cartilage and labral damage observed during surgical hip dislocation for stable slipped capital femoral epiphysis. J Pediatr Orthop. 2010;30:26-30.

33. Slongo T, Kakaty D, Krause F, Ziebarth K. Treatment of slipped capital femoral epiphysis with a modified Dunn procedure. $J$ Bone Joint Surg Am. 2010;92:2898-2908.

34. Souder CD, Bomar JD, Wenger DR. The role of capital realignment versus in situ stabilization for the treatment of slipped capital femoral epiphysis. J Pediatr Orthop. 2014;34:791-798.

35. Southwick WO. Osteotomy through the lesser trochanter for slipped capital femoral epiphysis. J Bone Joint Surg Am. 1967;49:807-835.

36. Southwick WO. Slipped capital femoral epiphysis. J Bone Joint Surg Am. 1984;66:1151-1152.

37. Wensaas A, Gunderson RB, Svenningsen S, Terjesen T. Femoroacetabular impingement after slipped upper femoral epiphysis: the radiological diagnosis and clinical outcome at long-term follow-up. J Bone Joint Surg Br. 2012;94:1487-1493.

38. Ziebarth K, Leunig M, Slongo T, Kim YJ, Ganz R. Slipped capital femoral epiphysis: relevant pathophysiological findings with open surgery. Clin Orthop Relat Res. 2013;471:2156-2162.

39. Ziebarth K, Zilkens C, Spencer S, Leunig M, Ganz R, Kim YJ. Capital realignment for moderate and severe SCFE using a modified Dunn procedure. Clin Orthop Relat Res. 2009;467:704-716. 